\title{
Traditional Chinese medicine in the prevention and treatment of cancer and cancer metastasis (Review)
}

\author{
LIN YE ${ }^{1-3}$, YONGNING JIA ${ }^{1,2,4}, \mathrm{KE} \mathrm{JI}^{1-3}$, ANDREW J. SANDERS ${ }^{1-3}, \mathrm{KAN} \mathrm{XUE}^{4}$, \\ JIAFU JI ${ }^{4}$, MALCOLM D. MASON ${ }^{3}$ and WEN G. JIANG ${ }^{1-3}$ \\ ${ }^{1}$ Cardiff University-Peking University Cancer Institute; ${ }^{2}$ Metastasis and Angiogenesis Research Group; \\ ${ }^{3}$ Institute of Cancer and Genetics, Cardiff University School of Medicine, Cardiff CF14 4XN, UK; \\ ${ }^{4}$ Key Laboratory of Carcinogenesis and Translational Research (Ministry of Education), \\ Department of Gastrointestinal Surgery, Peking University Cancer Hospital and Institute, \\ Haidian, Beijing 100142, P.R. China
}

Received June 16, 2014; Accepted February 25, 2015

DOI: $10.3892 / \mathrm{ol} .2015 .3459$

\begin{abstract}
Traditional Chinese medicine (TCM) has been a major part of healthcare in China, and has extensively affected medicine and healthcare in surrounding countries over a long period of time. In the fight against cancer, certain anticancer remedies using herbs or herbal formulas derived from TCM have been developed for the management of malignancies. Furthermore, there are clinical trials registered for the use of herbal remedies in cancer management. Herbal medicine has been used as part of combined therapies to reduce the side-effects of chemotherapy, including bone marrow suppression, nausea and vomiting. Herbal remedies have also been used as chemopreventive therapies to treat precancerous conditions in order to reduce the incidence of cancer in high-risk populations. Emerging evidence has revealed that herbal remedies can regulate the proliferation, apoptosis, adhesion and migration of cancer cells. In addition to this direct effect upon cancer cells, a number of herbal remedies have been identified to suppress angiogenesis and therefore reduce tumour growth. The inhibition of tumour growth may also be due to modifications of the host immune system by the herbal treatment. However, the precise mechanisms underlying the therapeutic effects of herbal remedies remain poorly understood and are yet to be fully elucidated. The present study aims to summarize the current literature and clinical trial results of herbal remedies for cancer treatment, with a particular focus on the recent findings and development of the Yangzheng Xiaoji capsule.
\end{abstract}

Correspondence to: Dr Wen G. Jiang, Cardiff University-Peking University Cancer Institute, Henry Wellcome Building, Cardiff University School of Medicine, Heath Park, Cardiff CF14 4XN, UK E-mail: jiangw@cardiff.ac.uk

Key words: traditional Chinese medicine, therapy, cancer, metastasis

\section{Contents}

1. Introduction

2. Chinese herbal medicine in combined therapies for malignancies

3. Molecular and cellular machinery of TCM in the treatment and prevention of cancer

4. Targeting angiogenesis in solid tumours

5. Herbal therapy for precancerous disorders

6. Perspectives

\section{Introduction}

The use of complementary and alternative medicine (CAM) has been widely accepted as a therapeutic approach for the management of malignancies, particularly in the supportive and palliative care of cancer patients. In 2007, $\$ 33.9$ million was spent by adults in the USA on CAM (1). Furthermore, CAM is commonly used by cancer patients; in fact, $25-50 \%$ of prostate cancer patients have used at least one CAM modality (2). As a major part of CAM in Western countries, traditional Chinese medicine (TCM) has also been used to treat malignancies. In 2011, a series of correspondences concerning traditional Asian medicines were published, which created interest in investigating the potential use of traditional Asian medicines for cancer management, in particular, the study of relevant therapeutic approaches using current scientific research methods (3). Traditional medicines, including those practiced in China, Korea, Japan and other countries worldwide, may appear mystical and pseudoscientific due to a poor scientific understanding of their actions and a lack of convincing clinical evidence according to the standards of modern science and medicine. Success has been achieved in studies of traditional medicine, including artemisinin for malaria and arsenic trioxide for leukaemia $(4,5)$. To date, the majority of traditional remedies lack the standardisation and scientific evidence required to validate their effect, efficiency and application. As part of traditional Asian medicines, TCM has been used to prevent and treat a number of disorders, with documentary 
evidence, for two thousand years. Over the last few decades, studies of TCM in cancer have focused on molecules extracted and purified from anticancer herbs. Icariside II, purified from the root of Epimedium koreanum Nakai, is able to induce the apoptosis of human acute myeloid leukemia U937 cells through the STAT3 pathway (6). Artemisinin, a compound extracted from the Chinese medical herb, Qing Hao, has been widely used in the treatment of malaria, and has also been involved in cancer treatment (7-10). Based upon the evidence from clinical studies, investigations of certain herbal remedies have been further developed into clinical trials to examine their efficacy and safety in cancer management. However, data concerning the effect and mechanisms of TCM in the prevention and treatment of malignancies, particularly anticancer remedies comprising multiple herbs, remain limited.

The Yangzheng Xiaoji (YZXJ) capsule, derived from a TCM formula, has exhibited anticancer actions (11). In a randomized double-blind lung cancer study, patients who received treatment with conventional chemotherapy combined with YZXJ ( $n=304)$ exhibited significantly increased levels of complete and partial disease remission compared with those who received chemotherapy alone $(n=103)(23.3$ vs. $14 \%$, respectively; $\mathrm{P}<0.01)(11)$. The patients who received YZXJ also presented with decreased bone marrow suppression. Previous studies have established that YZXJ is able to directly inhibit the adhesion and migration of cancer cells. In addition to its direct effect on cancer cells, it is also able to suppress the angiogenic capacities of vascular endothelial cells, which may contribute to the inhibitory effect on in vivo tumour growth (12-14).

Although progress has been made in the research and application of herbal remedies for the treatment of malignancies, data from clinical trials are still required in order to understand the mechanisms that underlie the action of these medicines and therefore guide their clinical application appropriately.

\section{Chinese herbal medicine in combined therapies for ma- lignancies}

At present, common treatments for cancer include surgery, chemotherapy, radiotherapy, immunotherapy and targeted therapy. Surgery remains the first treatment choice for the majority of early-stage solid tumours, whilst other treatments and surveillance may be used in the management of advanced-stage tumours, or as adjuvant therapies for less advanced tumours. The side-effects and toxicity of chemotherapy and radiotherapy often restrict their application and effectiveness. The damage these therapies cause to the normal tissues of patients is usually more severe than that to the tumours. Those patients who must undergo long courses of chemotherapy and radiotherapy may have to end treatment as a result of the accompanied toxicity and additional side-effects. In combination with chemotherapy and/or radiotherapy, TCM has the potential to be used as an assistant therapeutic approach; it can enhance the response rate (RR) to chemotherapy and radiotherapy, reduce the severity of toxic effects and other side-effects caused by chemotherapy and radiotherapy, and improve the quality of life and survival of cancer patients (15).
To date, a number of herbs have been reported to be beneficial to cancer patients. These include single herbs, traditional herbal formulations and Chinese medicine preparations. A number of single herbs have been individually adopted for cancer treatment, including Radix Astragali (Huang Qi) (16), ginseng (Ren Shen) (17), blister beetle (also known as Mylabris; Ban Mao) (18), toad venom (Chan Su) (19), garlic (Da Suan) (20) and turmeric (Jiang Huang) (21). Chinese traditional herbal formulations were usually developed by ancestors of TCM, and were based on Chinese medicine theories, including yin and yang, the five elements and meridians. A single formulation often has numerous components. At present, the formulations adopted for cancer treatment always contain certain common major components, which are the popular single herbs with anticancer activities.

Chinese medicine preparations are recently developed herbal remedies that are prepared from either single herb or traditional herbal formulations using modern advanced pharmaceutical technologies. There are a number of different types of preparations, including injections, tablets, pills, capsules and orally-administered liquids. Compared with traditional decoctions, Chinese medicine preparations are safer, more effective and easier to use. With the development of pharmacological research concerning herbal medicines, increasing numbers of effective ingredients have been successfully extracted and their efficacy clarified, which has promoted the development of Chinese medicine preparations. Mylabris has been identified to induce the apoptosis of cancer cells and promote functions of the immune system, but it is greatly restricted in its clinical application due to renal toxicity and bone marrow suppression (22). Subsequent studies have demonstrated that cantharidin is one of its effective ingredients and that a number of artificial analogues of cantharidin exhibit similar effects, but with lower toxicities $(23,24)$. Increased knowledge of herbal medicines will aid in the understanding of Chinese formulations and preparations for the treatment of cancer. The present review summarises the data on single herbs and Chinese medicine preparations that are widely adopted for the treatment of cancer.

The present review describes the commonly used single herbs, Radix Astragali (Huang Qi), ginseng (Ren Shen), Mylabris (Ban Mao) and toad venom (Chan Su), which according to preclinical studies and clinical trials, have anticancer activities. Garlic (Da Suan) and turmeric (Jiang Huang) have demonstrated marked anticancer activities in preclinical and clinical studies, but not in clinical trials (Table I). In addition to the single herbs, there are also certain Chinese medicine preparations being used to treat cancer.

Radix Astragali (Huang Qi). According to a number of pharmacological studies, Astragalus has a potential immunomodulatory role when combined with chemotherapy and radiotherapy; it promotes the activity of lymphocytes, natural killer cells and macrophages (16), which leads to the secretion of interleukin-6 (IL-6) and tumour necrosis factor (TNF). In addition, Astragalus can enhance the biological function of IL-2, whilst also simultaneously reducing its adverse effects $(25,26)$. In the most recent systematic review concerning the combination of Radix Astragali and platinum-based chemotherapy, 34 studies were selected from an initial 1,305, and quantified 
for subsequent meta-analysis. A reduced risk of mortality over $6,12,24$ and 36 months was reported by 7, 12, 9 and 6 of the studies, respectively, with a significantly increased tumour response observed in 30 studies (risk ratio, 1.34; 95\% confidence interval, 1.24-1.46; $\mathrm{P}<0.0001)$. Although the number of studies concerning chemotherapy-associated toxicity is relatively small, a significant reduction in grade III or IV white blood cell (WBC) toxicity and grade III or IV haemoglobin $\mathrm{Hb}$ toxicity was identified in patients who had received a combined therapy. An improved or stable performance status was also observed in the majority of the studies $(27,28)$.

Ginseng (Ren shen) and Mylabris (Ban Mao). In addition to Radix Astragali, ginseng and Mylabris are two other single herbs commonly used in combination with chemotherapy and radiotherapy. Ginseng is able to modulate the function of the immune system and reduce the side-effects of radiotherapy. Furthermore, it can dilate small arteries and veins, inhibit oxidation and suppress inflammation (17). According to these anticancer properties, gingseng is a potential candidate for cancer prevention and treatment. It has been used to suppress inflammation, and reduce cancer occurrence and recurrence in several types of tumours, including gastric, liver, pancreatic and colon cancers $(17,29)$. In addition, gingseng has the potential to reduce the toxicity of radiotherapy in normal human tissue, which may be due to its anti-oxidation and immune regulation effects. Cantharidin is the anticancer ingredient in Mylabris (23). In addition to functioning in immune system regulation, cantharidin and Mylabris are able to promote the apoptosis of cancer cells, particularly in liver and oesophageal cancers. However, their application in cancer treatment has been restricted by the occurrence of renal toxicity and bone marrow suppression. However, several cantharidin analogues have been synthesized that exhibit similar anticancer activities, but with lower toxicities (24).

In a systematic review of TCM treatment in hepatocellular cancers, 45 out of an initial 130 studies were included for meta-analysis. The single herbs included Radix Astragali, ginseng, beetle extract (Mylabris), toad skin secretions (bufotoxin), Atractylodes, Bupleurum and Curcuma. The pooled RR of the 37 studies indicated that formulations containing gingseng, Astragalus and Mylabris were significantly correlated with increased tumour responses, including complete response (CR), partial response (PR), stable disease and progressive disease. Among the 45 included studies, 15, 22, 4 and 15 reported a positive correlation between TCM usage and 6, 12, 18 and 24 months survival, respectively. However, just 7 out of the 45 included studies reported improvements in symptoms. There were 6, 4 and 4 studies that reported an improvement in the symptoms of abdominal pain, fatigue and poor appetite, respectively (30).

A further meta-analysis investigated the treatment of colorectal cancer with TCM in combination with the FOLFOX 4 regimen (comprising folinic acid, fluorouracil and oxaliplatin). The single herbs that were used included Radix Astragali, ginseng, Atractylodes macrocephala, Poria cocos, Coix lachryma-jobi and Sophora flavescens. In total, 13 out of an initial 1,487 studies were included. In this meta-analysis, tumour response was defined as a CR or PR. A significant improvement in tumour response was 
observed in the combined treatment group compared with the FOLFOX 4 only group. In terms of survival, a positive effect on overall survival was reported in 3 of the studies, but was not supported by the meta-analysis. Although there was a significant difference in favour of the group that received the TCM and FOLFOX 4 regimen, this was the result of only 3 studies. Karnofsky performance status was reported in 10 of the studies, and was significantly higher in the test groups in 9 of the studies, indicating an improved performance status of the test group. In addition, all 13 included studies reported alleviated chemotherapy toxicities. The total grade III or IV events for neutropenia, nausea, vomiting and neurotoxicity were also significantly fewer in the test groups (31).

Toad venom. Chan $\mathrm{Su}$ is another single herb with anticancer activity. Bufadienolides, including bufalin, cinobufagin, resibufogenin and telocinobufagin, are the major active constituents derived from Chan $\mathrm{Su}$ (32). Chan $\mathrm{Su}$ and its constituents have been reported to be able to suppress cellular growth and promote apoptosis in leukaemia, liver, lung and prostate cancers (19). The Chinese medicine preparation, Hua Chan Su, contains bufalin, resibufogenin and cinobufagin, which are derived from Chan $\mathrm{Su}(33,34)$. Hua Chan $\mathrm{Su}$ can induce the apoptosis and inhibit the growth of heptocellular carcinoma (HCC) cell lines (35-38). In clinical studies, Hua Chan $\mathrm{Su}$ has been identified to improve performance status in HCC, non-small cell lung cancer and pancreatic cancer patients (38). One of the constituents, cinobufagin, when used alone or in combination with other chemotherapy agents, has also demonstrated anticancer activities in HCC, non-small cell lung cancer, pancreatic cancer and gallbladder carcinoma patients (39). The combination of Hua Chan $\mathrm{Su}$ and chemotherapy has been used for the treatment of advanced gastric cancer. In total, 14/15 studies reviewed by Xie et al (40) indicated that the combination of Hua Chan Su and chemotherapy was unable to increase the tumour RR or 1-year survival rate. However, the performance status was improved by the combined therapy. Further analysis revealed that the combination of Hua Chan Su and chemotherapy significantly reduced the symptoms of chemotherapy-induced toxicity, including leucocytopenia, gastrointestinal side-effects and neurotoxicity (40).

Shenqi Fuzheng injection. The Shenqi Fuzheng injection is extracted from the herbal medicines, Radix Astragali (Huang Qi) and Codonopsis pilosula (Dang Shen). Astragalus has been identified to exhibit marked anticancer activities. Codonopsis pilosula has certain anticancer activities that are similar to those of gingseng. A promising anticancer effect may therefore exist in the Shenqi Fuzheng injection. In clinical trials, Shenqi Fuzheng improved the tumour response and reduced chemotherapy-induced toxicity in the combined treatment of advanced cancers, including lung, breast and colorectal cancers $(41,42)$. In addition, the Shenqi Fuzheng injection has been widely used in the treatment of non-small cell lung cancer. In a meta-analysis, 29 relevant clinical studies were selected, with 27 out of the 29 reporting a tumour RR. A significantly higher tumour RR was observed in the test group, which received Shenqi Fuzheng in addition to platinum-based chemotherapy. Furthermore, a significantly improved performance status was observed in the test group. The chemotherapy-associated side-effects reported in the meta-analysis included grade III or IV WBC, platelet (PLT) or HB toxicity, nausea and vomiting. Significant reductions in toxicity were also observed in the pooled RR for WBC, PLT and HB toxicity, nausea and vomiting (41).

Kang Laite (KLT) injection. The KLT injection is extracted from the Chinese herb, coixseed (coicis semen yokuinin). This Chinese medicine preparation has obtained approval from the Chinese government for the treatment of lung and hepatic cancer (43). It has been reported that KLT is able to inhibit cellular mitosis during the $\mathrm{G}_{2} / \mathrm{M}$ phase of the cell cycle and promote apoptosis via the Fas/FasL pathway (44). A phase I study indicated that there was no marked toxicity when the KLT injection was administered at the maximum dose of $50,000 \mathrm{mg} /$ day in the first cycle (45). KLT has been widely used in combination with chemotherapy for the treatment of non-small cell lung cancer. In a meta-analysis that included 26 studies, the pooled analysis of 22 of the studies indicated that the combination of KLT and chemotherapy increased the RR. An improvement in the performance status, in addition to a reduction in chemotherapy-induced adverse events, were also observed in the test group. Similar effects were evident in the pooled RR for leukopenia, anemia, thrombocytopenia, nausea, vomiting and phlebitis (46).

YZXJ capsule. The YZXJ capsule is a formula derived from the collateral disease theory. The capsule consists of 16 herbs, including Radix Astragali, ginseng, Atractylodes macrocephala koidz and Poria cocos (Table II) (47). Of these ingredients, Astragalus and Panax ginseng are important single herbs with anticancer activities. YZXJ has been used in the treatment of liver, breast, lung, colorectal and gastric cancers. Preclinical studies have revealed that it exhibits various anticancer activities, including inhibition of adhesion, migration and angiogenesis, and affects the expression of proliferating cell nuclear antigen, p53 and vascular endothelial growth factor (VEGF) $(13,14)$. Recently, its application in the treatment of various tumours was reviewed in a meta-analysis, which included 19 clinical studies. In this meta-analysis, YZXJ was revealed to be able to significantly reduce chemotherapy-induced toxicity, and the pooled odds ratio for bone marrow suppression, leukopenia, thrombocytopenia, gastrointestinal adverse reaction and hepatotoxicity (48).

Despite the promising results of the systematic reviews described in the present review, the majority of the studies have had certain limitations. Firstly, the sample sizes of these studies were relatively small, with 30-40 cases on average. Secondly, the Jadad scale quality scores of the studies were low, with the majority under 3 . Thirdly, few studies described the detailed procedure of randomisation. Fourthly, the included studies were not rigorously controlled; for example, the analysis of survival time required the authors to describe how the patients lost to follow up were handled, what percentage were lost to follow-up and whether these patients were censored in the analysis. Therefore, more rigorously controlled, randomized studies are required. However, given the fact that clinical trials consume money and time, 


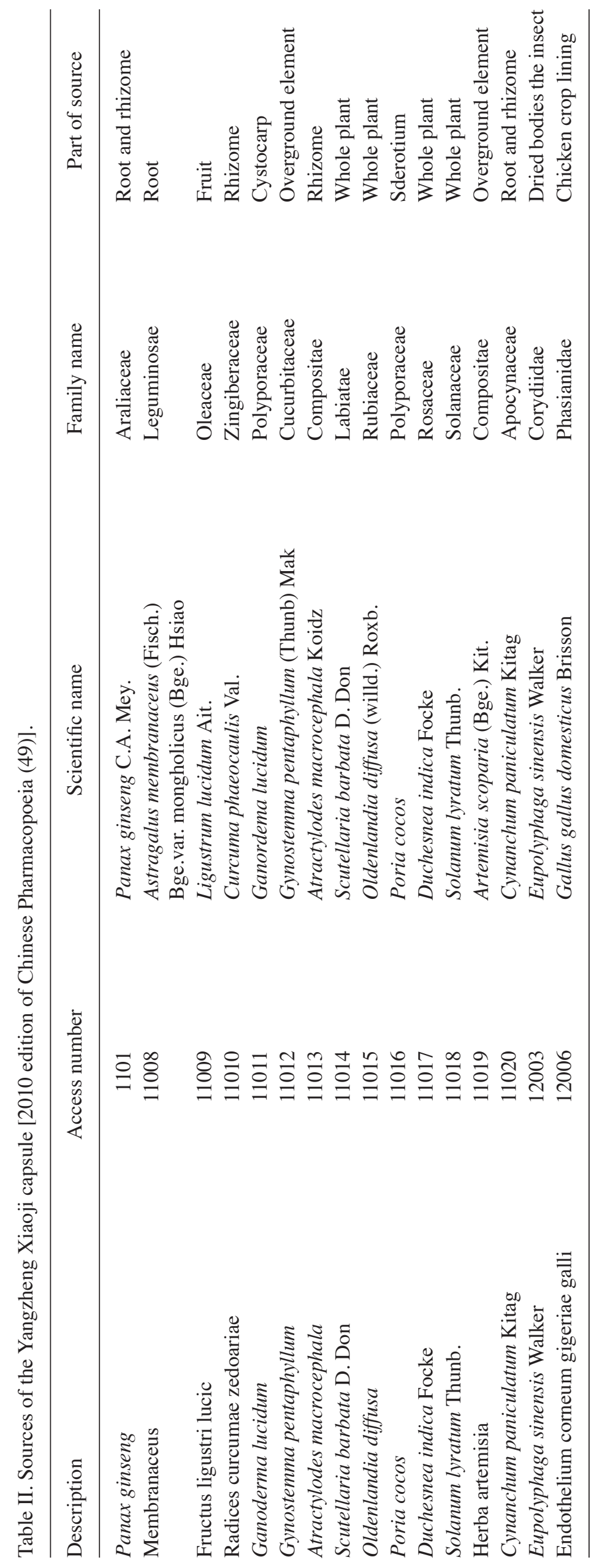


the included studies can at least identify herbal medicines worthy of further investigation.

\section{Molecular and cellular machinery of TCM in the treat- ment and prevention of cancer}

Clinical studies have demonstrated the benefits of certain herbs and herbal medications for the clinical management of malignancies. Studies have been performed in order to identify the mechanisms that underlie the therapeutic effects of these herbal remedies, but at present, have not been able to provide a solid scientific explanation. In the following section, the present review analyses the current literature in order to portray the current understanding of how herbal remedies affect cancer.

Effect of TCM on the immune system. It is believed that the immune system may be regulated by a number of different TCMs. During the development and progression of cancer, the adoptive and adaptive immune responses can be evaded by cancer cells. In addition, radiotherapy and chemotherapy can also impair the immune system, particularly through bone marrow suppression. Patients receiving radiotherapy and chemotherapy may benefit from herbal medicines due to their capacity to improve the functions of the immune system (16,24-26). Firstly, they are believed to enhance the immune response against the tumour. Secondly, they may suppress the immune inhibitory mechanisms and shift the immune system balance. Finally, they may restore the impaired immune system back to its normal condition following the administration of chemotherapy and radiotherapy.

Radix Astragali has been identified to increase interferon and TNF secretion, and activate lymphocytes, natural killer $(\mathrm{NK})$ cells and macrophages against tumours. In addition, Radix Astragali has been revealed to cooperate with IL-2 in order to stimulate lymphokine-activated killer cells against tumour cells $(16,24,25)$. Mylabris and the TJ-41 formulation have been reported to be able to restore antitumour T cells $(18,24)$. In one study concerning TJ-41, the oral administration of TJ-41 in tumour-bearing mice under a stress-induced immunosuppression state, restored the lymphoid cells and cytokine secretion, and in addition, activated cytotoxic T lymphocytes (CTLs). The underlying mechanisms in the stress burdened tumour-bearing mice included stimulated lymphocyte proliferation, restored serum corticosterone levels, recovered surface expression of co-stimulatory molecules, enhanced IL-12 secretion, suppressed IL-4 production, a shift in the TH1/TH2 balance to a TH-1 type response and the restoration of CTLs (50). The TJ-41 formulation has also been reported to enhance immunological function $(51,52)$. Another Chinese medicinal formula known as Juzen-Taiho-To (JTT) contains 10 single herbs and has been reported to reduce the metastatic potential of various solid tumours (53-55). One study reported that the oral administration of JTT could suppress the growth of mouse subcutaneous gliomas and increase the survival rate of the tumour-bearing mice. The potential underlying mechanisms may include the activation of NK cells and the enhanced secretion of TNF (53). Miyagami and Katayama (56) also reported that the administration of JTT had several effects, including a reduction in the number of suppressor $\mathrm{T}$ cells, the promotion of TNF- $\alpha$ secretion and the activation of NK cells. However, the number of cytotoxic $\mathrm{T}$ cells was reported to be unchanged.

TCM and the apoptosis of cancer cells. Cancer cell growth depends upon the balance of cell gain and loss. The cell cycle of cancer cells occurs at a much faster rate than that of normal cells, and ultimately leads to cancer cell accumulation. By contrast, cell loss via necrosis, apoptosis and autophagy are much slower. Certain TCMs may also induce cell cycle arrest or apoptosis, and therefore inhibit cell growth and metastasis. Garlic extracts are primarily composed of sulphur compounds, particularly allicin, diallyl sulphide (DAS), diallyl disulphide (DADS), diallyltrisulphide (DATS) and ajoene, all of which exhibit anticancer activities. The antiproliferative effects of the extracts are associated with the induction of apoptosis $(20,57)$. Morphological changes and DNA fragmentation have been observed in cells treated with DADs and DATs $(58,59)$. Furthermore, a previous study reported that DAS induced the apoptosis of solid tumour cells, according to the results of sub- $\mathrm{G}_{1}$ fraction and terminal deoxynucleotidyl transferase-mediated dUTP nick end labeling (60). Further research has revealed the mechanisms underlying DAS-induced apoptosis, including elevated p53 and p21/waf1 expression, and a shift in the B-cell lymphoma 2 (Bcl-2)/Bcl-2 associated X protein (Bax) balance (61).

The anticancer activity of cinobufacini (Hua Chan $\mathrm{Su}$ ) is primarily through the induction of apoptosis. In a previous study, the water-soluble constituent extracted from toad venom, cinobufacini, was revealed to suppress the growth of HCC HepG2 and Bel-7402 cell lines in a time- and concentration-dependent manner. The growth inhibition was due to the promotion of apoptosis through a mitochondrial pathway. Morphological changes, including chromatin condensation, and nuclear and cytoplasmic fragmentation, were clearly observed, particularly morphological changes in the disturbance of the transmembrane potential. In addition, increased Bax and decreased Bcl-2 expression was observed following cinobufacini treatment, which led to an increased $\mathrm{Bax} / \mathrm{Bcl}-2$ ratio. Furthermore, poly ADP ribose polymerase cleavage was observed together with cytochrome release and caspase- 3 and -9 activation (35).

TCM induces cell cycle arrest. Bu-Zhong-Yi-Qi-Tang (BZYQT) is a mixed formulation, with major constituents of astragaloside IV, ginsenoside Rb1 and Rg1, saikosaponin a and c, and glycyrrhizin. The effects of the formula on liver cancer Hep3B, HepG2 and HA22T cells were analysed in a previous study (62). The results of the study revealed that the whole mixture of BZYQT, or a mixture of the major compounds rather than any single constituent in the mixture, inhibited liver cancer cell growth, particularly in the Hep3B cells. The cell cycle $G_{0} / G_{1}$ ratio increased in proportion following treatment with BZYQT, and finally lead to $G_{0} / G_{1}$ arrest. In addition, apoptotic cells and evidence of DNA fragmentation were clearly observed. Therefore, BZYQT was established to inhibit hepatoma cell growth by inducing apoptosis through $\mathrm{G}_{0} / \mathrm{G}_{1}$ arrest.

A previous study described the effects of the Sho-Saiko-To formulation on a number of ovarian cell lines (63). In the study, all six ovarian cancer cell lines exhibited a response to 
Sho-Saiko-To. Glycyrrhizin, baicalin, and baicalein are three constituents of Sho-Saiko-To, and are all able to inhibit cancer cell growth (64-67). In another study, Sho-Saiko-To exerted similar activity, in which apoptosis was involved in the growth inhibitory effect at different concentrations; apoptosis was induced at a concentration of $1,000 \mu \mathrm{g} / \mathrm{ml}$, while cell death via necrosis occurred when the concentration increased to $5,000 \mu \mathrm{g} / \mathrm{ml}$. Although the precise underlying mechanism was unclear, one of the ingredients of Sho-Saiko-To, namely baicalein, was reported to be able to inhibit the activity of type II topoisomerases and induce the apoptosis of the cells (68). The Sho-Saiko-To mixture itself is able to inhibit hepatoma cell growth by inducing apoptosis and cell cycle arrest $(63,69)$.

Constituents of the garlic extract have also been identified to be actively involved in the cell cycle arrest of tumour cells. DATs and DADs are able to induce $\mathrm{G}_{2} / \mathrm{M}$ mitotic arrest. In addition, DATs have been demonstrated to induce the Ser(216) phosphorylation of $\mathrm{Cdc} 25 \mathrm{c}$, promote the generation of reactive oxygen species, and increase the cell division cycle inhibition of p21 (70), while DAPs have been established to inhibit p34cdc2 kinase activity and the growth of $\mathrm{H}$-ras oncogene-transformed tumours (71).

Cantharidin is the active component of Mylabris. Previous data has revealed that it inhibits certain PPP family phosphatases, which have antitumour activity, and promotes apoptosis in several cancer cell lines. Flow cytometry analysis revealed that cantharidin slowed the progression of the cell cycle following DNA replication. However, prior to apoptosis, $\mathrm{G}_{1}-\mathrm{S}$ or $\mathrm{S}-\mathrm{G}_{2}$ phase progression was not delayed. By contrast, cantharidin inhibited cell growth during $\mathrm{G}_{2}$ or early $\mathrm{M}$ phase. However, the cell cycle arrest occurred prior to the end of mitosis and was accompanied by the appearance of abnormal mitotic spindles. Using time-lapse microscopy, cantharidin was observed to interrupt chromosome location in metaphase, extend the mitotic arrest and lead to apoptosis rather than anaphase. Further suppression of phosphase PP2A $\alpha$, but not PP2A $\beta$, induced the arrest of metaphase. During this period, the chromosomes moved between the spindle and metaphase plate (72).

TCM regulates the adhesion and motility of cancer cells. Cancer cell adhesion and invasion are important steps in the process of cancer metastasis. While relatively few TCMs have been reported to be able to affect these steps, our institute (Cardiff University - Peking University Cancer Institute) has focused on metastasis and angiogenesis for decades, and has recently identified that the YZXJ formulation is effective in inhibiting cancer cell adhesion, migration and angiogenesis in vitro and in vivo (12-14). The YZXJ capsule consists of 16 herbs, including Panax ginseng, Astragalus, Ligustrum, Curcuma phaeocaulis and Ganoderma lucidum (Table II). Its extract, DME25, has not demonstrated any significant effect on the growth of cancer cells, but it does markedly suppress cell adhesion and migration. The results of an electric cell-substrate impedance sensing assay revealed that YZXJ inhibited the cell adhesion of gastric cancer HGC27 cells (in a concentration-dependent manner), colorectal cancer HRT18 cells, breast cancer MCF7 cells, lung cancer A549 cells and osteosarcoma MG-63 cells, and also the migration of the lung cancer and colorectal cancer cells (12). In addition, it was verified that the inhibitory effect of YZXJ on the adhesion of cancer cells was associated with the PI3K signalling pathway. Wortmannin, an inhibitor of the activity of PI3K, is able to suppress PI3K/AKT signalling and consequently reduce the adhesiveness of cancer cells. DME25, which also targets the AKT pathway, can enhance this inhibitory effect. The effect of DME25 on the PI3K pathway may not only depend upon the single AKT signalling pathway (13). The identification of the effect of DME25 on vascular endothelial cells demonstrated that YZXJ markedly suppressed the formation of the canaliculi of vascular endothelial cells, so that the cell matrix adhesion and migration was inhibited in a concentration-dependent manner. Cell matrix adhesion is an important indicator of cellular migration. In addition, cellular adhesion and migration are important during angiogenesis, particularly during canaliculus formation by vascular endothelial cells, when they adhere to the cell matrix and subsequently migrate into the extracellular matrix. Hence, the inhibitory effect of YZXJ on the adhesion and migration of vascular endothelial cells may be the principal mechanism for inhibiting canaliculus formation (14).

Previous studies have revealed that the focal adhesion kinase (FAK) signalling pathway is important for cell matrix adhesion and cell-extracellular matrix adhesion (73-75). Following the connection of integrins and matrix, a series of cellular events are triggered and activated, particularly the FAK signal pathway, in the interaction between cells and matrix (76). Subsequently, the interaction between integrins and the cell cytoskeleton system is triggered (76). It has been reported that DME25 exhibited an inhibitory effect on the phosphorylation of the FAK pathway, and that the addition of a FAK inhibitor significantly enhanced the effect of DME25 on the FAK pathway (77). In addition, it was revealed that YZXJ not only inhibited the growth of colorectal and lung cancer cells, but also suppressed the formation of mouse peritoneal tumour nodules in an in vivo study. The significant inhibition of tumour growth was observed following either the oral administration or intraperitoneal (i.p.) injection of YZXJ. The results of FAK and pFAK immunofluorescence colony staining revealed that YZXJ lowered the expression of FAK and almost inhibited its activation following the administration of DME25 in combination with a FAK inhibitor. Based on the study of the effect of YZXJ on the adhesion of tumour and mesothelial cells, a further study demonstrated that peritoneal tumour nodules treated with YZXJ through i.p. injection were significantly decreased compared with those of the untreated control group (unpublished experimental data). Therefore, on the basis of the results from previous studies, which demonstrated that YZXJ was involved in the adhesion between tumour cells and mesothelial cells, and in tumour cell migration and angiogenesis through the analysis of an in vivo model, it can be hypothesized that YZXJ has an inhibitory effect on peritoneal metastasis.

\section{Targeting angiogenesis in solid tumours}

The process of angiogenesis, whereby new blood vessels develop from pre-existing ones, is important during normal development and homeostasis. Angiogenesis is a key process involved in a number of physiological events, including wound 
healing, development, reproduction and growth. Under normal circumstances, this process is tightly regulated, however, abnormal levels or poor control are apparent in a number of different disease states, including cancer. Angiogenesis is important for the progression of cancer and the development of tumours where, without an independent blood supply, tumour size is limited by its reliance on simple diffusion in order to obtain resources and remove metabolic waste. This has long been realised, and has lead to the development and implementation of anti-angiogenic strategies for the treatment of cancer $(78,79)$. As discussed in the present review, the use of traditional medicines or herbal extracts in the treatment of diseases, such as cancer, has been long established and practiced. Scientific scrutiny of these medicines and extracts has begun to demonstrate their efficacy in important physiological processes, such as angiogenesis, and therefore highlight their importance as treatment regimes.

TCM/herbs implicated in tumour angiogenesis. The mechanisms through which various herbal medicines exert their anticancerous roles have begun to be elucidated. In addition to their direct effect on cancer cells, a number of herbal medicines have also been demonstrated to exhibit an impact upon tumour progression through their effects on angiogenesis. One such remedy displaying anti-angiogenic effects is the standardised Ginkgo biloba extract, EGb $761^{\circledR}$. Extracts from G. biloba have demonstrated inhibitory effects or chemopreventative properties in vitro/in vivo in ovarian cancer (80), breast cancer (81), hepatocellular carcinoma (82) and oral cavity cancer (83) cells. A study by Koltermann et al (84) identified an anti-angiogenic role for the standardised extract of G. biloba, EGb 761. In this study, a marked anti-proliferative effect of EGb 761 on endothelial cells was observed, in addition to a significant inhibition of endothelial cell migration and tubule formation on Matrigel following treatment with EGb 761. These anti-angiogenic in vitro effects were mimicked in vivo, where the induction of angiogenesis in a chorioallantois membrane assay was inhibited following EGb 761 treatment (84). A role was established for EGb 761 in enhancing the activity of tyrosine phosphatases, including SHP-1, which in turn prevented signal transduction through the Raf/MEK/ERK pathway in response to growth factor stimulation (fibroblast growth factor or VEGF) (84).

Scutellaria barbata D. Don is another herb frequently used in TCM. Extracts from $S$. barbata have demonstrated inhibitory effects on a number of cancer cells in vitro (85-87). The therapeutic potential of extracts from this herb have been further demonstrated through a phase $1 \mathrm{~B}$ dose elevation clinical trial for an orally delivered aqueous extract, BZL101, in metastatic breast cancer patients, in which it was revealed to be well tolerated and demonstrated promising clinical evidence (88).

Extracts from this herb have also exhibited anti-angiogenic roles. In a recent study by Dai et al (88), the potential of the total flavonoids of $S$. barbata, TF-SB, to inhibit angiogenesis in vitro and in vivo were analysed. In this study, TF-SB was identified to have dose-dependent inhibitory effects on the proliferation, migration and tubule formation of human umbilical vein endothelial cells in vitro. Similar effects were observed in vivo using a chorioallantois membrane assay, whereby treatment with TF-SB significantly decreased the number of blood vessels formed compared with the control groups. The study further established that TF-SB treatment affected the expression of and decreased the levels of VEGF, a trend that was also correlated with the treatment concentration (89). This result is consistent with that of an earlier study, which revealed an inhibitory effect of $S$. barbata extract on endothelial cell migration and proliferation (90). This study also demonstrated that $S$. barbata significantly decreased the transcriptional activity of hypoxia inducible factor-1 $\alpha$ and the expression of VEGF in lung cancer cells, delayed the tumour burden, and reduced the capillary density in an in vivo model (90). An ethanol extract of S. barbata has also proven to be effective in reducing tumour volume in colorectal xenograph mice, a finding that was accompanied by a reduction in tumour microvessel density (91). The treatment of mice with this extract also reduced the expression of a number of molecules associated with the sonic hedgehog ( $\mathrm{SHH}$ ) signalling pathway in tumour tissues, and downregulated the expression of VEGF-A and VEGF receptor 2, which indicated that the inhibition in colorectal cancer cell growth in vivo following treatment with $S$. barbata may be due to its inhibitory effects on SHH-mediated angiogenesis (91). Quercetin is a type of flavonoid that is generally extracted from the G. biloba leaf it reduces the viability of vascular endothelial cells and the formation of tubules, in which the ERK pathway may be involved (92).

YZXJ in tumour angiogenesis. A previous study assessed the efficacy of the TCM formula, YZXJ, for its potential to affect the angiogenesis profile (14). In this study, the impact of YZXJ on key angiogenic traits, including cell growth, matrix attachment, migration and tubule formation, were examined in the human endothelial HECV cell line. YZXJ was identified to have an inhibitory effect on endothelial tubule formation, and significantly inhibited this process at sub-cytotoxic concentrations (dilution, 1:1,000) compared with untreated control cells. Similarly, YZXJ was also identified to inhibit endothelial cell migration, another important process involved in the angiogenic cascade, in a concentration-dependent manner, and to significantly inhibit cell-matrix attachment. Therefore, this study demonstrated an anti-angiogenic role for YZXJ in these model systems. A further study was also conducted in order to investigate the mechanistic side of these effects and to identify potential associations with FAK. The inhibitory effects of YZXJ on cellular migration and matrix adhesion were synergistically increased following the inhibition of FAK, bringing about stronger inhibition of these key processes when YZXJ treatment was combined with a small molecule FAK inhibitor. Furthermore, treatment with YZXJ, whilst not having a substantial impact on FAK staining intensity, substantially reduced the staining intensity of p-FAK upon immunofluorescence staining analysis. p-FAK staining was further decreased through a combination of YZXJ treatment and FAK inhibition. Furthermore, western blot analysis demonstrated a decrease in tyrosine-phosphorylated FAK in HECV cells that had been treated with YZXJ. These results identified an anti-angiogenic role for YZXJ, and suggested that this may be induced, at least in part, by its impact on the FAK signalling pathway (14).

Anti-angiogenic therapies have marked potential for the treatment of cancer. A number of these therapies are currently implemented and demonstrate efficacy in the treatment of various cancers (93). The potential of herbal extracts and TCMs to exhibit anti-angiogenic properties, together with their ability to affect tumour cell function directly, further highlights the 
importance and potential of these remedies in the development of novel therapeutic strategies to target cancer progression.

\section{Herbal therapy for precancerous disorders}

Cancer may develop from the progression of precancerous lesions and conditions. Precancerous lesions, for example, high-grade squamous intraepithelial lesions caused by infection with human papillomaviruses (HPVs) 16, 18, 45, 31 and 33, can develop into squamous cell carcinoma, whilst another strain of HPV, HPV 18, predominates in adenocarcinomas (94). Precancerous conditions or premalignant conditions, including actinic keratosis, Barrett's oesophagus, atrophic gastritis and oral submucous fibrosis, are generally considered to possess a significantly increased risk of cancer. Two decades ago, investigators began to examine the preventative effects of herbal formulas on different precancerous conditions. An anticancer 2 tablet, composed of six TCM herbs, was assessed for the treatment of epithelial dysplasia in the cheek pouches of hamsters that had been exposed to a carcinogen (95). Radix curcumae is one of the commonly used TCM herbs for the treatment of malignancies. Its extract is able to reduce precancerous lesions of the stomach induced by N-methyl-N'-nitro-N-nitrosoguanidine (96). Herbal supplements, consisting of quercetin, curcumin, silymarin, ginseng and rutin, that are added to the diet can be beneficial to patients with precancerous lesions of the large intestine (97). This herbal mixture has been shown to suppress aberrant crypt foci in an azoxymethane-induced rat colon cancer model, and also to induce apoptosis via the regulation of Bax (proapoptotic) and Bcl-2 (anti-apoptotic), ultimately leading to capase-9 activation. Clinical trials using Zeng Sheng Ping (ZSP) to treat oesophageal epithelial dysplasia have been performed in two Chinese districts with a high risk of oesophageal carcinoma $(98,99)$. The results from these clinical trials have revealed the benefits of ZSP, which can reduce the incidence of cancer in high-risk groups with severe oesophageal epithelium dysplasia by $34.5-47.79 \%$ over a follow-up period of up to 9 years. A further study demonstrated that ZSP exhibited chemopreventive effects in patients with oral leukoplakia, and also affected tumour development in two animal models of oral cancer (100). YZXJ has also been reported to be able to improve atypical dysplasia of the stomach (47). There have been a number of clinical studies that have established effects on precancerous conditions or lesions. However, the quality of these clinical studies was such that they were unable to validate the findings and therefore, further clinical trials are warranted. In addition to the requirement for improved clinical studies, the underlying mechanisms, for example, the effect on oncogenes, tumour suppressor genes and key pathways involved in tumourigenesis are yet to be investigated.

\section{Perspectives}

Increasing evidence has demonstrated that herbal remedies from TCM have the potential to be developed into therapeutic approaches for the clinical management of various malignancies. The corresponding effects and benefits include its ability to act as an effective therapy for certain malignancies, reduced side-effects when combined with chemotherapy/radiotherapy, a chemopreventive effect for certain precancerous conditions/lesions, improved quality of life and prolonged survival. However, a number of limitations and challenges exist that reduce the progress of studies on the use of TCM remedies for the treatment of cancers. These limitations include the liability and variability of the herbal medications, a lack of reliable biomarkers for clinical and laboratory-based studies, a lack of relevant pharmacokinetic data for standardising dosage, and a lack of reliable data from well-designed clinical studies to further establish subsequent clinical trials. For example, one previous clinical trial was terminated following the contamination of a patented mixture of eight herbs with warfarin and indomethacin (101). An improvement in the set-up of clinical studies is critical in order to provide solid evidence for subsequent clinical trials. The Best Case Series programme from the National Cancer Institute has provided a standardised process to validate results using rigorous scientific methods, particularly for the studies of conventional medicine $(102,103)$. In addition, a requirement exists to consider the side-effects and toxicities of herbal medications (104) in the evaluation of any clinical studies.

\section{References}

1. Nahin RL, Barnes PM, Stussman BJ and Bloom B:Costs of complementary and alternative medicine (CAM) and frequency of visits to CAM practitioners: United States, 2007. Natl Health Stat Report: 1-14, 2009.

2. Klempner SJ and Bubley G: Complementary and alternative medicines in prostate cancer: from bench to bedside? Oncologist 17: 830-837, 2012.

3. Grayson M: Traditional Asian Medicine. Nature 480: S81, 2011.

4. Chen GQ, Zhu J, Shi XG, et al: In vitro studies on cellular and molecular mechanisms of arsenic trioxide (As2O3) in the treatment of acute promyelocytic leukemia: As2O3 induces NB4 cell apoptosis with downregulation of Bcl-2 expression and modulation of PML-RAR alpha/PML proteins. Blood 88: 1052-1061, 1996.

5. Cai XZ: Observation of therapeutic effect of single-dose combined administration of Qinghaosu, sulphomethoxine, pyrimethamine and primaquine in the treatment of chloroquine-resistant malignant malaria (author's transl). Zhonghua Nei Ke Za Zhi 20: 724-727, 1981 (In Chinese).

6. Kang SH, Jeong SJ, Kim SH, et al: Icariside II induces apoptosis in U937 acute myeloid leukemia cells: role of inactivation of STAT3-related signaling. PLoS One 7: e28706, 2012.

7. He R, Mott BT, Rosenthal AS, Genna DT, Posner GH and Arav-Boger R: An artemisinin-derived dimer has highly potent anti-cytomegalovirus (CMV) and anti-cancer activities. PLoS One 6: e24334, 2011.

8. Firestone GL and Sundar SN: Anticancer activities of artemisinin and its bioactive derivatives. Expert Rev Mol Med 11: e32, 2009.

9. Lai H, Nakase I, Lacoste E, Singh NP and Sasaki T: Artemisinin-transferrin conjugate retards growth of breast tumors in the rat. Anticancer Res 29: 3807-3810, 2009.

10. Sun WC, Han JX, Yang WY, Deng DA and Yue XF: Antitumor activities of 4 derivatives of artemisic acid and artemisinin B in vitro. Zhongguo Yao Li Xue Bao 13: 541-543, 1992 (In Chinese).

11. Zhang SY, Gu CH and Gao XD: A randomly double-blinded and multicentre study of chemotherapy assisted Yangzhengxiaoji capsule on treating primary hepatic carcinoma. J Diffic Compl Case 8: 461-464, 2009

12. Jiang WG, Ye L, Ji K, et al: Antitumour effects of Yangzheng Xiaoji in human osteosarcoma: the pivotal role of focal adhesion kinase signalling. Oncol Rep 30: 1405-1413, 2013.

13. Ye L, Ji K, Frewer N, Ji J and Jiang WG: Impact of Yangzheng Xiaoji on the adhesion and migration of human cancer cells: the role of the AKT signalling pathway. Anticancer Res 32: 2537-2543, 2012.

14. Jiang WG, Ye L, Ji K, Frewer N, Ji J and Mason MD: Inhibitory effects of Yangzheng Xiaoji on angiogenesis and the role of the focal adhesion kinase pathway. Int J Oncol 41: 1635-1642, 2012.

15. Konkimalla VB and Efferth T: Evidence-based Chinese medicine for cancer therapy. J Ethnopharmacol 116: 207-210, 2008. 
16. No authors listed: Astragalus membranaceus. Monograph. Altern Med Rev 8: 72-77, 2003.

17. Hofseth LJ and Wargovich MJ: Inflammation, cancer, and targets of ginseng. J Nutr 137 (Suppl 1): 183S-185S, 2007.

18. Wang GS: Medical uses of Mylabris in ancient China and recent studies. J Ethnopharmacol 26: 147-162, 1989.

19. Lu CX, Nan KJ and Lei Y: Agents from amphibians with anticancer properties. Anticancer Drugs 19: 931-939, 2008.

20. Shukla Y and Kalra N: Cancer chemoprevention with garlic and its constituents. Cancer Lett 247: 167-181, 2007.

21. Kuttan R, Bhanumathy P, Nirmala K and George MC: Potential anticancer activity of turmeric (Curcuma longa). Cancer Lett 29: 197-202, 1985.

22. Wang GS: Medical uses of mylabris in ancient China and recent studies. J Ethnopharmacol 26: 147-162, 1989.

23. Lee TK, Johnke RM, Allison RR, O'Brien KF and Dobbs LJ Jr: Radioprotective potential of ginseng. Mutagenesis 20 237-243, 2005.

24. Liu D and Chen Z: The effects of cantharidin and cantharidin derivates on tumour cells. Anticancer Agents Med Chem 9: 392-396, 2009

25. Yoshida Y, Wang MQ, Liu JN, Shan BE and Yamashita U: Immunomodulating activity of Chinese medicinal herbs and Oldenlandia diffusa in particular. Int J Immunopharmacol 19 359-370, 1997.

26. Wang Y, Qian XJ, Hadley HR and Lau BH: Phytochemicals potentiate interleukin-2 generated lymphokine-activated killer cell cytotoxicity against murine renal cell carcinoma. Mol Biother 4: 143-146, 1992.

27. Liu J, Shi Z, Xu Z, et al: Clinical observation on treatment of non-parvicellular carcinoma of the lung with jin fu kang oral liquid. J Tradit Chin Med 20: 96-100, 2000

28. McCulloch M, See C, Shu XJ, et al: Astragalus-based Chinese herbs and platinum-based chemotherapy for advanced non-small-cell lung cancer: meta-analysis of randomized trials. J Clin Oncol 24: 419-430, 2006.

29. Yun TK and Choi SY: Preventive effect of ginseng intake against various human cancers: a case-control study on 1987 pairs. Cancer Epidemiol Biomarkers Prev 4: 401-408, 1995.

30. Wu P, Dugoua JJ, Eyawo O, Mills EJ: Traditional Chinese Medicines in the treatment of hepatocellular cancers: a systematic review and meta-analysis. J Exp Clin Cancer Res 28: 112, 2009.

31. Chen M, May BH, Zhou IW, Xue CC and Zhang AL: FOLFOX combined with herbal medicine for advanced colorectal cancer: a systematic review. Phytother Res 28: 976-991, 2014.

32. Wang Z, Wen J, Zhang J, Ye M and Guo D: Simultaneous determination of four bufadienolides in human liver by high-performance liquid chromatography. Biomed Chromatogr 18: 318-322, 2004

33. Wang J, Jin Y, Xu Z, Zheng Z and Wan S: Involvement of caspase-3 activity and survivin downregulation in cinobufocini-induced apoptosis in A 549 cells. Exp Biol Med (Maywood) 234: 566-572, 2009.

34. Wang DL, Qi FH, Xu HL, et al: Apoptosis-inducing activity of compounds screened and characterized from cinobufacini by bioassay-guided isolation. Mol Med Rep 3: 717-722, 2010

35. Qi FH, Li AY, Lv H, et al: Apoptosis-inducing effect of cinobufacini, Bufo bufo gargarizans Cantor skin extract, on human hepatoma cell line BEL-7402. Drug Discov Ther 2: 339-343, 2008.

36. Qi F, Li A, Zhao L, et al: Cinobufacini, an aqueous extract from Bufo bufo gargarizans Cantor, induces apoptosis through a mitochondria-mediated pathway in human hepatocellular carcinoma cells. J Ethnopharmacol 128: 654-661, 2010.

37. Cui X, Inagaki Y, Xu H, et al: Anti-hepatitis B virus activities of cinobufacini and its active components bufalin and cinobufagin in HepG2.2.15 cells. Biol Pharm Bull 33: 1728-1732, 2010

38. Meng Z, Yang P, Shen Y, et al: Pilot study of Huachansu in patients with hepatocellular carcinoma, non-small cell lung cancer, or pancreatic cancer. Cancer 115: 5309-5318, 2009.

39. Qin TJ, Zhao XH, Yun J, Zhang LX, Ruan ZP and Pan BR: Efficacy and safety of gemcitabine-oxaliplatin combined with huachansu in patients with advanced gallbladder carcinoma. World J Gastroenterol 14: 5210-5216, 2008.

40. Xie X, Huang X, Li J, et al: Efficacy and safety of Huachansu combined with chemotherapy in advanced gastric cancer: a meta-analysis. Med Hypotheses 81: 243-250, 2013.

41. Dong J, Su SY, Wang MY and Zhan Z: Shenqi fuzheng, an injection concocted from Chinese medicinal herbs, combined with platinum-based chemotherapy for advanced non-small cell lung cancer: a systematic review. J Exp Clin Cancer Res 29: 137, 2010
42. Dai Z, Wan X, Kang H, et al: Clinical effects of shenqi fuzheng injection in the neoadjuvant chemotherapy for local advanced breast cancer and the effects on T-lymphocyte subsets. J Tradit Chin Med 28: 34-38, 2008

43. Yang AK, He SM, Liu L, Liu JP, Wei MQ and Zhou SF: Herbal interactions with anticancer drugs: mechanistic and clinical considerations. Cur Med Chem 17: 1635-1678, 2010.

44. Lu Y, Wu LQ, Dong Q and Li CS: Experimental study on the effect of Kang-Lai-Te induced apoptosis of human hepatoma carcinoma cell HepG2. Hepatobiliary Pancreat Dis Int 8: 267-272, 2009

45. Guo HY, Cai Y, Yang XM, et al: Randomized phase II trial on mitomycin-C/cisplatin +/- KLT in heavily pretreated advanced breast cancer. Am J Chin Med 36: 665-674, 2008.

46. Liu X, Xu F, Wang G, Diao X and Li Y: Kanglaite injection plus chemotherapy versus chemotherapy alone for non-small cell lung cancer patients: A systematic review and meta-analysis. Curr Ther Res Clin Exp 69: 381-411, 2008.

47. Wang QL, Xue CM, Wu SP, Li YX and Bi XJ: Clinical observation on Yangzhengxiaoji capsule in treatment of 60 cases of moderate to severe degree atypical proliferation of gastric mucosa. Chin J Diffic Compl Case 7: 38-39, 2008 (In Chinese).

48. Xue K, Shan F and Ji J: Meta-analysis of the safety of Yangzhengxiaoji capsule for the treatment of cancer and precancerosis. Chin J Clin Oncol 40: 1318-1323, 2013.

49. Chinese Pharmacopoeia Commission: Pharmacopoeia of the People's Republic of China 2010. Vol 1.9th edition. Chemical Industry Press, Beijing, 2011.

50. Li T, Tamada $\mathrm{K}$, Abe $\mathrm{K}$, et al: The restoration of the antitumor $\mathrm{T}$ cell response from stress-induced suppression using a traditional Chinese herbal medicine Hochu-ekki-to (TJ-41:Bu-Zhong-Yi-Qi-Tang). Immunopharmacology 43: 11-21, 1999.

51. Niwa K, Hashimoto M, Morishita S, et al: Preventive effects of Juzen-taiho-to on N-methyl-N-nitrosourea and estradiol-17beta-induced endometrial carcinogenesis in mice. Carcinogenesis 22: 587-591, 2001.

52. Kamiyama $\mathrm{H}$, Takano S, Ishikawa E, Tsuboi $\mathrm{K}$ and Matsumura $\mathrm{A}$ : Anti-angiogenic and immunomodulatory effect of the herbal medicine 'Juzen-taiho-to' on malignant glioma. Biol Pharm Bull 28: 2111-2116, 2005.

53. Saiki I: A Kampo medicine 'Juzen-taiho-to' - prevention of malignant progression and metastasis of tumor cells and the mechanism of action. Biol Pharm Bull 23: 677-688, 2000.

54. Ohnishi Y, Fujii H, Hayakawa Y, et al: Oral administration of a Kampo (Japanese herbal) medicine Juzen-taiho-to inhibits liver metastasis of colon 26-L5 carcinoma cells. Jpn J Cancer Res 89: 206-213, 1998

55. Matsumoto M, Seya T, Kikkawa S, et al: Interferon gamma-producing ability in blood lymphocytes of patients with lung cancer through activation of the innate immune system by BCG cell wall skeleton. Int Immunopharmacol 1: $1559-1569,2001$

56. Miyagami M and Katayama Y: Improvement of host-immunity by adjuvant therapy with juzen-taiho-to for patients with brain tumors. No Shinkei Geka 31: 401-409, 2003 (In Japanese).

57. Ngo SN, Williams DB, Cobiac L and Head RJ: Does garlic reduce risk of colorectal cancer? A systematic review. J Nutr 137: 2264-2269, 2007.

58. Sundaram SG and Milner JA: Diallyl disulfide inhibits the proliferation of human tumor cells in culture. Biochim Biophys Acta 1315: 15-20, 1996.

59. Nishino $H$, Iwashima A, Itakura $Y$, Matsuura $H$ and Fuwa $T$ : Antitumor-promoting activity of garlic extracts. Oncology 46: 277-280, 1989.

60. Arora A and Shukla Y: Induction of apoptosis by diallyl sulfide in DMBA-induced mouse skin tumors. Nutr Cancer 44: 89-94, 2002.

61. Arora A, Siddiqui IA and Shukla Y: Modulation of p53 in 7,12-dimethylbenz[a]anthracene-induced skin tumors by diallyl sulfide in Swiss albino mice. Mol Cancer Ther 3: 1459-1466, 2004

62. Kao ST, Yeh CC, Hsieh CC, et al: The Chinese medicine Bu-Zhong-Yi-Qi-Tang inhibited proliferation of hepatoma cell lines by inducing apoptosis via G0/G1 arrest. Life Sci 69: 1485-1496, 2001

63. Zhu K, Fukasawa I, Furuno M, et al: Inhibitory effects of herba drugs on the growth of human ovarian cancer cell lines through the induction of apoptosis. Gynecol Oncol 97: 405-409, 2005.

64. Okita K, Li Q, Murakamio T and Takahashi M: Anti-growth effects with components of Sho-saiko-to (TJ-9) on cultured human hepatoma cells. Eur J Cancer Prev 2: 169-175, 1993. 
65. Chang WH, Chen $\mathrm{CH}$ and Lu FJ: Different effects of baicalein, baicalin and wogonin on mitochondrial function, glutathione content and cell cycle progression in human hepatoma cell lines. Planta Med 68: 128-132, 2002.

66. Chang WH, Chen CH, Gau RJ, et al: Effect of baicalein on apoptosis of the human Hep G2 cell line was induced by mitochondrial dysfunction. Planta Med 68: 302-306, 2002.

67. Motoo Y and Sawabu N: Antitumor effects of saikosaponins, baicalin and baicalein on human hepatoma cell lines. Cancer Lett 86: 91-95, 1994

68. Matsuzaki Y, Kurokawa N, Terai S, Matsumura Y, Kobayashi N and Okita K: Cell death induced by baicalein in human hepatocellular carcinoma cell lines. Jpn J Cancer Res 87: 170-177, 1996.

69. Yano H, Mizoguchi A, Fukuda K, et al: The herbal medicine sho-saiko-to inhibits proliferation of cancer cell lines by inducing apoptosis and arrest at the G0/G1 phase. Cancer Res 54: 448-454, 1994.

70. Xiao D, Herman-Antosiewicz A, Antosiewicz J, et al: Diallyl trisulfide-induced G(2)-M phase cell cycle arrest in human prostate cancer cells is caused by reactive oxygen species-dependent destruction and hyperphosphorylation of Cdc 25 C. Oncogene 24: 6256-6268, 2005.

71. Singh SV: Impact of garlic organosulfides on p21(H-ras) processing. J Nutr 131: 1046S-1048S, 2001.

72. Bonness K, Aragon IV, Rutland B, Ofori-Acquah S, Dean NM and Honkanen RE: Cantharidin-induced mitotic arrest is associated with the formation of aberrant mitotic spindles and lagging chromosomes resulting, in part, from the suppression of PP2Aalpha. Mol Cancer Ther 5: 2727-2736, 2006.

73. Kohno T, Matsuda E, Sasaki H and Sasaki T: Protein-tyrosine kinase CAKbeta/PYK2 is activated by binding $\mathrm{Ca} 2+/$ calmodulin to FERM F2 alpha2 helix and thus forming its dimer. Biochem J 410: 513-523, 2008.

74. Gilmore AP and Romer LH: Inhibition of focal adhesion kinase (FAK) signaling in focal adhesions decreases cell motility and proliferation. Mol Biol Cell 7: 1209-1224, 1996.

75. Cai J, Parr C, Watkins G, Jiang WG and Boulton M: Decreased pigment epithelium-derived factor expression in human breast cancer progression. Clin Cancer Res 12: 3510-3517, 2006.

76. Braren R, Hu H, Kim YH, Beggs HE, Reichardt LF and Wang R: Endothelial FAK is essential for vascular network stability, cell survival, and lamellipodial formation. J Cell Biol 172: $151-162,2006$

77. Ochiai K, Takita S, Eiraku T, et al: Phosphodiesterase inhibitors. Part 3: Design, synthesis and structure-activity relationships of dual PDE3/4-inhibitory fused bicyclic heteroaromatic-dihydropyridazinones with anti-inflammatory and bronchodilatory activity. Bioorg Med Chem 20: 1644-1658, 2012.

78. Folkman J: Angiogenesis: an organizing principle for drug discovery? Nat Rev Drug Discov 6: 273-286, 2007.

79. Potente M, Gerhardt $\mathrm{H}$ and Carmeliet P: Basic and therapeutic aspects of angiogenesis. Cell 146: 873-887, 2011.

80. Ye B, Aponte M, Dai Y, et al: Ginkgo biloba and ovarian cancer prevention: epidemiological and biological evidence. Cancer Lett 251: 43-52, 2007.

81. Papadopoulos V Kapsis A, Li H, et al: Drug-induced inhibition of the peripheral-type benzodiazepine receptor expression and cell proliferation in human breast cancer cells. Anticancer Res 20 : 2835-2847, 2000.

82. Chao JC and Chu CC: Effects of Ginkgo biloba extract on cell proliferation and cytotoxicity in human hepatocellular carcinoma cells. World J Gastroenterol 10: 37-41, 2004.

83. Kim KS, Rhee KH, Yoon JH, Lee JG, Lee JH and Yoo JB: Ginkgo biloba extract (EGb 761) induces apoptosis by the activation of caspase-3 in oral cavity cancer cells. Oral Oncol 41: 383-389, 2005

84.Koltermann A, Liebl J, Fürst R, Ammer H, Vollmar AM and Zahler S: Ginkgo biloba extract EGb 761 exerts anti-angiogenic effects via activation of tyrosine phosphatases. J Cell Mol Med 13: 2122-2130, 2009
85.Tang PM, Chan JY, Zhang DM, et al: Pheophorbide a, an active component in Scutellaria barbata, reverses $\mathrm{P}-$ glycoprotein-mediated multidrug resistance on a human hepatoma cell line R-HepG2. Cancer Biol Ther 6: 504-509, 2007.

86. Goh D, Lee YH and Ong ES: Inhibitory effects of a chemically standardized extract from Scutellaria barbata in human colon cancer cell lines, LoVo. J Agric Food Chem 53: 8197-8204, 2005

87. Yin X, Zhou J, Jie C, Xing D and Zhang Y: Anticancer activity and mechanism of Scutellaria barbata extract on human lung cancer cell line A549. Life Sci 75: 2233-2244, 2004.

88. Perez AT, Arun B, Tripathy D, et al: A phase 1B dose escalation trial of Scutellaria barbata (BZL101) for patients with metastatic breast cancer. Breast Cancer Res Treat 120: 111-118, 2010.

89.Dai ZJ, Lu WF, Gao J, et al: Anti-angiogenic effect of the total flavonoids in Scutellaria barbata D. Don. BMC Complement Altern Med 13: 150, 2013.

90. Shiau AL, Shen YT, Hsieh JL, Wu CL and Lee CH: Scutellaria barbata inhibits angiogenesis through downregulation of HIF-1 $\alpha$ in lung tumor. Environ Toxicol 29: 363-370, 2014.

91. Wei L, Lin J, Xu W, et al: Scutellaria barbata D. Don inhibits tumor angiogenesis via suppression of hedgehog pathway in a mouse model of colorectal cancer. Int J Mol Sci 13: 9419-9430, 2012

92.Zhao D, Qin C, Fan X, Li Y and Gu B: Inhibitory effects of quercetin on angiogenesis in larval zebrafish and human umbilical vein endothelial cells. Eur J Pharmacol 723: 360-367, 2014.

93. Bellou S, Pentheroudakis G, Murphy C and Fotsis T: Antiangiogenesis in cancer therapy: Hercules and hydra. Cancer Lett 338: 219-228, 2013.

94.Bosch FX and de Sanjosé S: The epidemiology of human papillomavirus infection and cervical cancer. Dis Markers 23 213-227, 2007.

95.Zhang KH and Xie JF: Experimental study on the treatment of oral precancerous lesion - short and long term effect of tablet Anticancer II. Ann Acad Med Singapore 18: 528-532, 1989.

96.Lu B, Xu L, Yu L and Zhang L: Extract of radix curcumae prevents gastric cancer in rats. Digestion 77: 87-91, 2008.

97. Volate SR, Davenport DM, Muga SJ and Wargovich MJ: Modulation of aberrant crypt foci and apoptosis by dietary herbal supplements (quercetin, curcumin, silymarin, ginseng and rutin). Carcinogenesis 26: 1450-1456, 2005.

98. Hou J, Lin PZ, Chen ZF, et al: Field population-based blocking treatment of esophageal epithelia dysplasia. World J Gastroenterol 8: 418-422, 2002.

99.Ding Z, Gao F and Lin P: Long-term effect of treating patients with precancerous lesions of the esophagus. Zhonghua Zhong Liu Za Zhi 21: 275-277, 1999 (In Chinese)

100. Sun Z, Guan X, Li N, Liu X and Chen X: Chemoprevention of oral cancer in animal models, and effect on leukoplakias in human patients with ZengShengPing, a mixture of medicinal herbs. Oral Oncol 46: 105-110, 2010.

101.Oh WK, Kantoff PW, Weinberg V, et al: Prospective, multicenter, randomized phase II trial of the herbal supplement, PC-SPES, and diethylstilbestrol in patients with androgen-independent prostate cancer. J Clin Oncol 22: 3705-3712, 2004

102. Olaku O, Zia F, Santana JM and White JD: The National Cancer Institute best case series program: a summary of cases of cancer patients treated with unconventional therapies in India. Integr Cancer Ther 12: 385-392, 2013.

103.Zia FZ and White JD: National Cancer Institute Best Case Series program. Integr Cancer Ther 8: 113-114, 2009.

104. Olaku O and White JD: Herbal therapy use by cancer patients: a literature review on case reports. Eur J Cancer 47: 508-514, 2011. 